\title{
Ranking different factors influencing flight delay
}

\author{
Meysam Kazemi Asfe ${ }^{a^{*}}$, Majid Jangi Zehi ${ }^{b}$, Mohammad Nabi Shahiki Tash ${ }^{\mathrm{c}}$ and Noor Mohammad \\ Yaghoubi $^{\text {d }}$
}

\author{
${ }^{a}$ Industrial Engineering Group, Zahedan Branch, Islamic Azad University, Zahedan, Iran \\ ${ }^{b}$ Member of Young Researchers Club, Computer Group, Khash Branch, Islamic Azad University, Khash, Iran \\ ${ }^{c}$ Economic Group, University of Sistan and Baluchestan, Zahedan, Iran \\ ${ }^{d}$ Management Group, University of Sistan and Baluchestan, Zahedan, Iran

\section{H R O N I C L E}

\section{A B S T R A C T}

Article history:

Received January 14, 2014

Accepted 10 June 2014

Available online

June 162014

Keywords:

Airline industry

Flight interruption

Flight delay

Analytical hierarchy process
Flight interruption is one of the most important issues in today's airline industry. Every year, most airlines spend significant amount of money to compensate flight delays. Therefore, it is important to detect important factors influencing on flight delays. This paper presents an empirical investigation to determine important factors on this issue. The study also asks some decision makers to make pairwise comparison and ranks various factors using the art of analytical hierarchy process. The study determines that technical defects and delayed entry were among the most important factors to blame for flight delays. In addition, announcing the postponement, replacement aircraft and path replacement are among the most important decisions facing managers in the aviation industry during the disruption of the flight.

\section{Introduction}

Flight interruption is one of the most important issues in today's airline industry. Every year, most airlines spend significant amount of money to compensate flight delays (Yao \& Jiandong, 2008; Churchill et al., 2010). Therefore, it is important to detect important factors on flight delays. There are many studies on the consequences of flight delays in the world. Airline flight schedules are very sensitive to individual flight delays because operating resources are linked together. Among the connective resources influenced by delayed flight operations are crews, aircraft passengers and gate space. In order to make an assessment on initial flight delays on an airline operating schedule Beatty et al. (1999) proposed the concept of a Delay Multiplier. They analyzed the relationship between the duration of initial delay, the time of day of the delay happening and airline schedule connectivity were applied to calculate the value of the delay multiplier. 
Schaefer and Millner (2001) presented a Detailed Policy Assessment Tool (DPAT) analysis to demonstrate the impacts of simulated changes in capacity due to inclement weather. Wang et al. (2003) presented a simple analytic model, which explicitly separates the controllable factors that impact delays and propagation of delays in the National Airspace System (NAS) from those items in a given scenario. They showed how the model could be implemented to better understand delay propagation from specific NAS airports, especially the impacts of flight schedule parameters on measured delay. Recorded data from actual NAS operations were implemented to derive forecast on key model parameters and to demonstrate how delay characteristics vary among various airports.

Hsu et al. (2007) built models to analyze flight-delay propagation, allowing for behavioral response. It studied three scenarios: the same aircraft operating consecutive flights, consecutive flights with different aircraft using the same gate, and various flights using various aircraft involving the transfers. The study further applied data from China Airline to show the application of the models. They reported that the parameter representing the behavioral response was close to or exceeds 1, stating that most of the buffer time was for consolidating more passengers into each flight. Moreover, the departure delay of flights was larger when considering the impacts of the time slot, than without considering the time slot.

In spite of recent decreases in air traffic, congestion is still at higher levels than it was many years ago. Because of this congestion and expected increases in traffic, strategic planning for daily operations in the air traffic system plays essential role. Weather typically has a strong impact in development of such plans, as it leads to degraded airport and airspace capacity, which in turn causes delays.

\section{The proposed study}

This paper presents an empirical investigation to determine important factors on flight delays. The study also asks some decision makers to make pairwise comparison and ranks various factors using the art of analytical hierarchy process. Fig. 1 demonstrates the hierarchy of the proposed study.

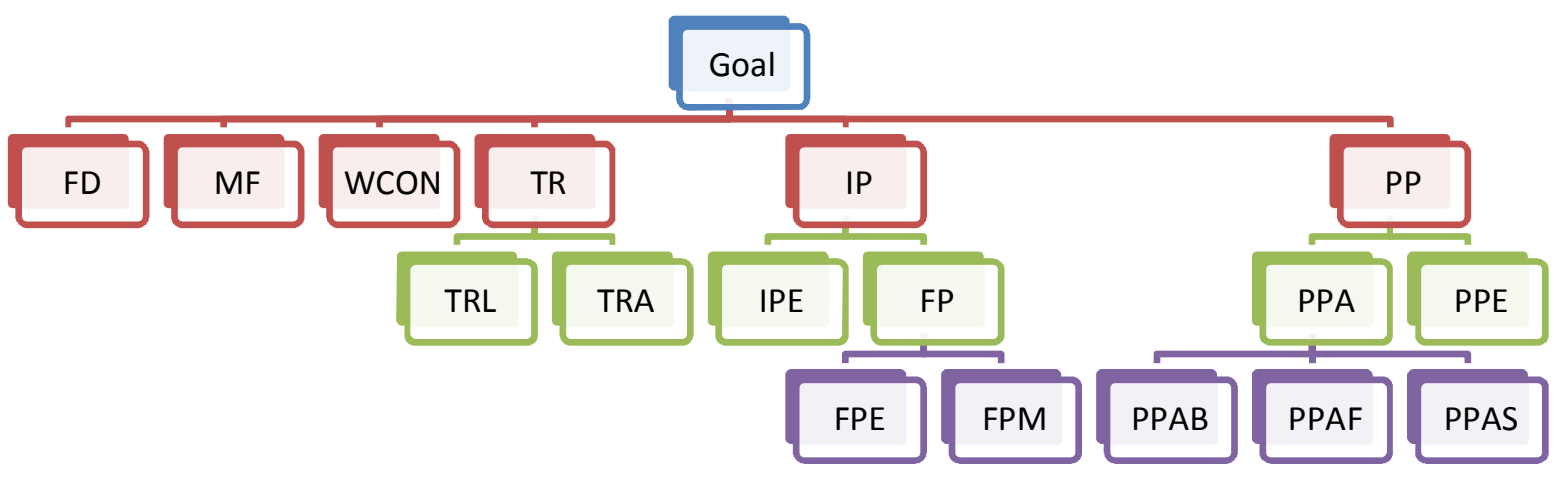

Fig. 1. The hierarchy of different factors

As we can observe from Fig. 1, there are three levels on the hierarchy of decision making procedure. In the first level, there are six factors including flight delay (FD), Malfunction (MF) Weather conditions (WCON), Airline traffic (TR), internal problems (IP) and Particular problems (PP). Three items including Airline traffic (TR), internal problems (IP) and Particular problems setup the second level of hierarchy. The Airline traffic (TR) consists of two factors including landing traffic (TRL) as well as air traffic (TRA). Internal problems includes two levels of employee related problem (IPE) and Fleet problems(FP), which is divided into two factors of external (FPE) and maintenance (FPM). 
Particular problem (PP) includes two levels of administration (PPA) and ending schedule (PPE). The administration related factors (PPA) includes three items including before takeoff (PPAB), fuel injection problems (PPAF) and security related problems (PPAS). Finally, Fleet problem includes two issues of embargo (FPE) and maintenance related issues (FPM). There are four alternatives of delayed announcement (DA), Flight Cancelation (FC), Flight replacement (FR) and route replacement (RR).

\section{The results}

In this section, we present details of the implementation of the analytical hierarchy process (AHP) (Saaty, 1988, 1990) to rank various factors in Iranian airline industry. During the past few years, AHP has become a popular technique for ranking various alternatives (Smith \& Tighe, 2006). Yoo and Choi (2006), for instance, used AHP for identifying relative importance of factors to improve passenger security checks at airports. Table 1 demonstrates the results of weights of the first level,

Table 1

The priorities of the first level

\begin{tabular}{ccccccc}
\hline & FD & MF & WCON & TR & IP & PP \\
\hline Priority & 0.262 & 0.293 & 0.143 & 0.069 & 0.192 & 0.041 \\
\hline
\end{tabular}

As we can observe from the results of Table 1, Malfunction is number one priority followed by flight delay, airline traffic, and weather conditions. Similarly, Table 2 demonstrates the priorities of the second level.

Table 2

The priorities of the second level

\begin{tabular}{cccccccc}
\hline & & & & Criteria & & Final \\
\hline Alternative & FD & MF & WCON & TR & IP & PP & Rank \\
\hline Delayed announcement & 0.611 & 0.354 & 0.173 & 0.415 & 0.340 & 0.514 & 0.353 \\
Flight Cancelation & 0.067 & 0.113 & 0.215 & 0.130 & 0.380 & 0.241 & 0.313 \\
Flight replacement & 0.190 & 0.485 & 0.045 & 0.087 & 0.080 & 0.123 & 0.139 \\
Route replacement & 0.132 & 0.048 & 0.567 & 0.367 & 0.200 & 0.122 & 0.195 \\
\hline
\end{tabular}

As we can observe from the results of Table 2, delayed announcement must be considered as the highest priority for handling flight delays followed by flight cancelation, route replacement and finally flight replacement must be offered as final remedy for compensation.

\section{Conclusion}

In this paper, we have presented an empirical investigation to determine important factors influencing flight delay in an Iranian airway industry. The proposed study has detected different factors and using analytical hierarchy process, the study has prioritized important actions when a flight delay occurs. Based on the results of our survey, in case of delay on flight schedule, airport managers must make a delay announcement on special billboard trying to keep the customers informed about the event and in case the problem insists, the flight needs to be cancelled and the event must be informed to all customers. The management may also look for route replacement and in case of absence of any alternative solution; customers have to be able to reimburse their expenses accordingly.

\section{Acknowledgment}

The authors would like to thank the anonymous referees for constructive comment on earlier version of this paper. 


\section{References}

Beatty, R., Hsu, R., Berry, L., \& Rome, J. (1999). Preliminary evaluation of flight delay propagation through an airline schedule. Air Traffic Quarterly, 7, 259-270.

Churchill, A. M., Lovell, D. J., \& Ball, M. O. (2010). Flight delay propagation impact on strategic air traffic flow management. Transportation Research Record: Journal of the Transportation Research Board, 2177(1), 105-113.

Hsu, C. I., Hsu, C. C., \& Li, H. C. (2007). Flight-delay propagation, allowing for behavioural response. International journal of critical infrastructures, 3(3), 301-326.

Saaty, T. L. (1988). What is the analytic hierarchy process? (pp. 109-121). Springer Berlin Heidelberg.

Saaty, T. L. (1990). How to make a decision: the analytic hierarchy process. European journal of operational research, 48(1), 9-26.

Schaefer, L., \& Millner, D. (2001). Flight delay propagation analysis with the detailed policy assessment tool. In Systems, Man, and Cybernetics, 2001 IEEE International Conference on (Vol. 2, pp. 1299-1303). IEEE.

Smith, J. T., \& Tighe, S. L. (2006). Analytic hierarchy process as a tool for infrastructure management. Transportation Research Record: Journal of the Transportation Research Board, 1974(1), 3-9.

Wang, P. T., Schaefer, L. A., \& Wojcik, L. A. (2003). Flight connections and their impacts on delay propagation. In Digital Avionics Systems Conference, 2003. DASC'03. The 22nd (Vol. 1, pp. 5-B). IEEE.

Yao, R., \& Jiandong, W. (2008). Design and implementation of web service to predict flight delay. Computer Engineering, 34(22), 66-69.

Yoo, K. E., \& Choi, Y. C. (2006). Analytic hierarchy process approach for identifying relative importance of factors to improve passenger security checks at airports. Journal of Air Transport Management, 12(3), 135-142. 